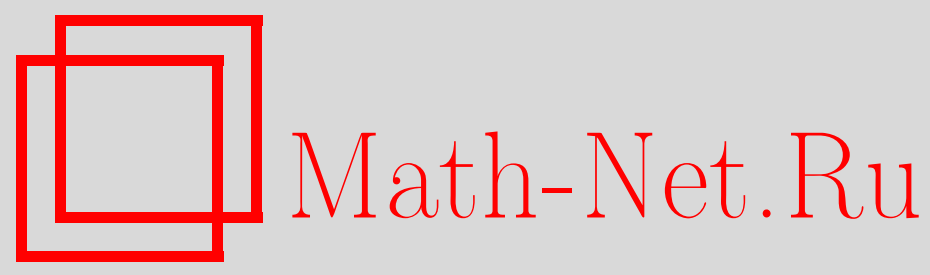

О. Ю. Шведов, О квазиклассических теориях поля, симметричных относительно группы Ли, Матем. заметки, 2003, том 73, выпуск 3, 474-477

DOI: https://doi.org/10.4213/mzm623

Использование Общероссийского математического портала Math-Net.Ru подразумевает, что вы прочитали и согласны с пользовательским соглашением http://www . mathnet.ru/rus/agreement

Параметры загрузки:

IP : 54.237 .59 .107

26 апреля 2023 г., $14: 23: 33$

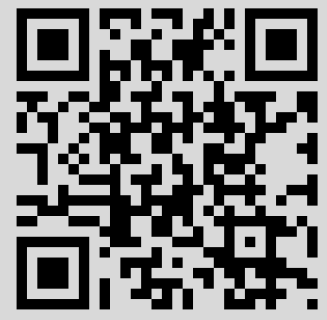




\section{О КВАЗИКЛАССИЧЕСКИХ ТЕОРИЯХ ПОЛЯ, СИММЕТРИЧНЫХ ОТНОСИТЕЛЬНО ГРУППЫ ЛИ}

\section{О. Ю. Шведов}

1. Асимптотические решения эволюционных уравнений, зависящих от малого параметра, во многих случаях выражаются [1] в каждый момент времени через элемент $X$ некоторого гладкого многообразия $\mathscr{X}$ ("расширенного фазового пространства") и элемента $f \in \mathscr{F}_{X}$ некоторого предгильбертова пространства $\mathscr{F}_{X}$, зависящего, вообще говоря, от $X$. Например, приближенные решения уравнения

$$
i \varepsilon \frac{\partial \psi}{\partial t}=H\left(x,-i \varepsilon \frac{\partial}{\partial x}\right) \psi, \quad x \in \mathbb{R}^{n}, \quad t \in \mathbb{R},
$$

построенные с помощью теории комплексного ростка Маслова [2] в точке, выражаются через зависящие от $t$ функции $S^{t} \in \mathbb{R}, P^{t}, Q^{t} \in \mathbb{R}^{n}$ и зависящую от $t \in \mathbb{R}$ и $\xi \in \mathbb{R}^{n}$ функцию $f^{t}(\xi)$, $f^{t} \in \mathscr{S}\left(\mathbb{R}^{n}\right):$

$$
\psi^{t}(x)=e^{\frac{i}{\varepsilon} S^{t}} e^{\frac{i}{\varepsilon} P^{t}\left(x-Q^{t}\right)} f^{t}\left(\frac{x-Q^{t}}{\sqrt{\varepsilon}}\right) .
$$

В данном случае $\mathscr{X}=\{(S, P, Q)\}=\mathbb{R}^{2 n+1}, \mathscr{F} X=\mathscr{S}\left(\mathbb{R}^{n}\right)$. Поскольку начальные данные для $(S, P, Q, f)$ однозначно определяют $\left(S^{t}, P^{t}, Q^{t}, f^{t}\right)$ [2], определены преобразования эволюции

$$
\begin{gathered}
u_{t}:\left(S^{0}, P^{0}, Q^{0}\right) \in \mathscr{X} \mapsto\left(S^{t}, P^{t}, Q^{t}\right) \in \mathscr{X}, \\
U_{t}\left(u_{t} X \leftarrow X\right): f^{0} \in \mathscr{F}_{X} \mapsto f^{t} \in \mathscr{F}_{u_{t} X} .
\end{gathered}
$$

Общие свойства асимптотических решений абстрактных уравнений могут быть исследованы $\mathrm{c}$ помощью понятия абстрактного канонического оператора [3], [4]. Совокупность $(X, f)$ рассматривается как точка на некотором ("квазиклассическом") векторном расслоении $(\mathscr{Z}, \mathscr{X}, \pi)$ : гладкое многообразие $\mathscr{X}$ является базой расслоения, а пространства $\mathscr{F}_{X}$ - слоями. Переходя к пополнению, можно считать пространства $\mathscr{F}_{X}$ полными. При этом преобразование (3) является автоморфизмом расслоения.

Часто встречаются динамические системы, инвариантные не только относительно эволюции: релятивистская и калибровочная инвариантность являются основополагающими аксиомами квантовой теории поля [5]. Сформулируем соответствующие определения для квазиклассических теорий.

ОПРЕДЕЛЕНИЕ 1. 1. Будем говорить, что на расслоении $(\mathscr{Z}, \mathscr{X}, \pi)$ действует калибровочная группа $\mathscr{L}$, если для каждого $\alpha \in \mathscr{L}$ задана совокупность $\lambda_{\alpha}: \mathscr{X} \rightarrow \mathscr{X}, V_{\alpha}\left(\lambda_{\alpha} X \leftarrow X\right)$ : $\mathscr{F}_{X} \rightarrow \mathscr{F}_{\lambda_{\alpha} X}$ гладкого отображения $\lambda_{\alpha}$ и унитарных преобразований $V_{\alpha}\left(\lambda_{\alpha} X \leftarrow X\right)$, определяющая автоморфизм расслоения $(\mathscr{Z}, \mathscr{X}, \pi)$, причем

$$
\begin{gathered}
\lambda_{\alpha_{1}} \lambda_{\alpha_{2}}=\lambda_{\alpha_{1} \alpha_{2},} \\
V_{\alpha_{1}}\left(\lambda_{\alpha_{1}} \lambda_{\alpha_{2}} X \leftarrow \lambda_{\alpha_{2}} X\right) V_{\alpha_{2}}\left(\lambda_{\alpha_{2}} X \leftarrow X\right)=V_{\alpha_{1} \alpha_{2}}\left(\lambda_{\alpha_{1}} \lambda_{\alpha_{2}} X \leftarrow X\right) .
\end{gathered}
$$

2. Две точки $\left(X_{1}, f_{1}\right)$ и $\left(X_{2}, f_{2}\right)$ пространства расслоения $\mathscr{Z}$ назьваются калибровочно әквивалентными, $\left(X_{1}, f_{1}\right) \sim\left(X_{2}, f_{2}\right)$, если для некоторого $\alpha \in \mathscr{L}$

$$
\lambda_{\alpha} X_{1}=X_{2}, \quad V_{\alpha}\left(X_{2} \leftarrow X_{1}\right) f_{1}=f_{2} .
$$

Работа выполнена при финансовой поддержке Российского фонда фундаментальных исследований, гранты № 02-01-1062 и № 02-01-06080. 
ОПРЕДЕЛЕНИЕ 2. Будем говорить, что на квазиклассическом расслоении $\pi: \mathscr{Z} \rightarrow \mathscr{X}$ с калибровочной группой $\mathscr{L}$ действует группа $G$, если

1) при всех $g \in G$ заданы отображения $u_{g}: \mathscr{X} \rightarrow \mathscr{X}$, причем

а) отображения $(g, X) \mapsto u_{g} X$ гладкие;

б) при любых $\alpha \in \mathscr{L}$ и $g \in G u_{g} \lambda_{\alpha} u_{g}^{-1}=\lambda_{\beta}$ для некоторого $\beta \in \mathscr{L}$, непрерывно зависящего от $\alpha, g$;

в) при любых $g_{1}, g_{2} \in G u_{g_{1}} u_{g_{2}}=\lambda_{\gamma} u_{g_{1}} g_{2}$ для некоторого $\gamma \in \mathscr{L}$, непрерьвно зависящего от $g_{1}, g_{2}$;

2) при всех $g \in G$ и $X \in \mathscr{X}$ заданы унитарные операторы $U_{g}\left(u_{g} X \leftarrow X\right): \mathscr{F}_{X} \rightarrow \mathscr{F}_{u_{g} X}$, причем

a) отображение $(g, h, \alpha) \mapsto U_{g}\left(u_{h} \lambda_{\alpha} X \leftarrow u_{g^{-1}} u_{h} \lambda_{\alpha} X\right)$ сильно непрерьвно по отношению к $(g, h, \alpha)$;

б) справедливы свойства

$$
\begin{gathered}
U_{g}\left(u_{g} \lambda_{\alpha} X \leftarrow \lambda_{\alpha} X\right) V_{\alpha}\left(\lambda_{\alpha} X \leftarrow X\right) U_{g^{-1}}\left(X \leftarrow u_{g} X\right)=V_{\beta}\left(u_{g} \lambda_{\alpha} X \leftarrow u_{g} X\right), \\
U_{g}\left(u_{g_{1}} u_{g_{2}} X \leftarrow u_{g_{2}} X\right) U_{g_{2}}\left(u_{g_{2}} X \leftarrow X\right) \\
=V_{\beta}\left(\lambda_{\beta} u_{g_{1} g_{2}} X \leftarrow u_{g_{1} g_{2}} X\right) U_{g_{1} g_{2}}\left(u_{g_{1} g_{2}} X \leftarrow X\right) .
\end{gathered}
$$

ЗАмечаниЕ. Данное определение означает, что каждому элементу $g \in G$ сопоставлен автоморфизм квазиклассического расслоения $\check{U}_{g}:(X, f) \mapsto\left(u_{g} X, U_{g}\left(u_{g} X \leftarrow X\right) f\right)$. При этом вместо точного равенства требуется калибровочная эквивалентность точек $\check{U}_{g_{1} g_{2}}(X, f)$ и $\check{U}_{g_{1}} \check{U}_{g_{2}}(X, f)$. Требуется также, чтобы калибровочно эквивалентные состояния переходили в калибровочно эквивалентные.

2. В сообщении рассматриваются инфинитезимальные свойства квазиклассических калибровочных преобразований. Законы композиции (5), (6) не очень удобны для исследования. Для приведения их к групповому виду рассмотрим преобразования сечений расслоения $\pi: \mathscr{Z} \rightarrow \mathscr{X}$. Будем говорить, что сечение $\Psi=\left\{\Psi_{Y} \in \mathscr{F}_{Y}, Y \in \mathscr{X}\right\}$ калибровочно инвариантно, если

$$
\Psi_{\lambda_{\alpha} Y}=V_{\alpha}\left(\lambda_{\alpha} Y \leftarrow Y\right) \Psi_{Y} .
$$

Определим отображение $\mathscr{U}_{g}$ как

$$
\left(\mathscr{U}_{g} \Psi\right)_{Y}=U_{g}\left(Y \leftarrow u_{g^{-1}} Y\right) \Psi_{u_{g^{-1}} Y} .
$$

Свойство (5) означает, что калибровочно инвариантные сечения переходят в калибровочно инвариантные, свойство (6) означает, что $\mathscr{U}_{g_{1} g_{2}} \Psi=\mathscr{U}_{g_{1}} \mathscr{U}_{g_{2}} \Psi$ на калибровочно инвариантньх сечениях $\Psi$. Аналогичные соображения для операторов эволюции рассматривались в [6].

Вместо сечений расслоения $(\mathscr{Z}, \mathscr{X}, \pi)$ удобнее рассматривать сечения подрасслоений $\left(Z_{[\bar{X}]}\right.$, $[\bar{X}], \pi)$ с $[\bar{X}]=\left\{\lambda_{\alpha} u_{g} \bar{X} \mid g \in G, \alpha \in \mathscr{L}\right\}$ и $Z_{[\bar{X}]}=\pi^{-1}[\bar{X}]$.

Обозначим через $G_{0}(\bar{X})$ множество всех $h \in G$ таких, что $u_{h} \bar{X}=\lambda_{\alpha} \bar{X}$ для некоторого $\alpha \in \mathscr{L}$. Будем говорить, что сильно непрерывное сечение $\Psi_{Y}$ подрасслоения $\left(Z_{[\bar{X}]},[\bar{X}], \pi\right)$ принадлежит классу $C_{0}([\bar{X}])$, если оно калибровочно инвариантно, а множество всех правых смежных классов $\bar{g} \in G / G_{0}(\bar{X})$ таких, что $\Psi_{\lambda_{\alpha} u \bar{g}} \bar{X} \neq 0$, предкомпактно на $G / G_{0}(\bar{X})$.

Введем в $C_{0}([\bar{X}])$ норму

$$
\|\Psi\|_{[\bar{X}]}=\sup _{X \in[\bar{X}]}\left\|\Psi_{X}\right\| .
$$

Обозначим через $C([\bar{X}])$ пополнение $C_{0}([\bar{X}])$ по данной норме.

Определим отображение $\mathcal{U}_{g}^{[\bar{X}]}$ по формуле (8).

Сопоставим каждой комплексной непрерывной ограниченной функции $\alpha_{X}, X \in \mathscr{X}$, на базе расслоения $\mathscr{X}$ оператор $v_{[\bar{X}]}[\alpha]: C_{0}([\bar{X}]) \rightarrow C_{0}([\bar{X}])$ вида $\left(v_{[\bar{X}]}[\alpha] \Psi\right)_{u_{h}} \bar{X}=\alpha_{u_{h}} \bar{X} \Psi_{u_{h}} \bar{X}$, которьй однозначно продолжается по непрерывности на $C([\bar{X}])$. Обозначим также $\left(w_{g} \alpha\right)_{X}=\alpha_{u_{g-1} X}$. Будем назьвать функцию $\alpha$ калибровочно инвариантной, если $\alpha_{\lambda_{\gamma} X}=\alpha_{X}$ при всех $\gamma \in \mathscr{L}$. 
Теорема 1. Пусть группа Ли $G$ действует на квазиклассическом расслоении $(\mathscr{Z}, \mathscr{X}, \pi)$ с калибровочной группой $\mathscr{L}$. Тогда оператор $\mathscr{U}_{g}^{[\bar{X}]}(8)$ :

- переводит $C_{0}([\bar{X}])$ в $C_{0}([\bar{X}])$;

- сохраняет норму (9);

- однозначно продолжается по непрерывности на $C([\bar{X}])$;

- удовлетворяет групповому свойству

$$
\mathcal{u}_{g_{1}}^{[\bar{X}]} \mathcal{U}_{g_{2}}^{[\bar{X}]}=\mathscr{U}_{g_{1} g_{2}}^{[\bar{X}]}, \quad \mathscr{U}_{e}^{[\bar{X}]}=1
$$

- сильно непрерывен по $g$ при $g=e$.

Для калибровочно инвариантных функиий $\alpha$ справедливо свойство

$$
\mathscr{U}_{g}^{[\bar{X}]} v_{[\bar{X}]}[\alpha] \Psi=v_{[\bar{X}]}\left[w_{g} \alpha\right] \mathscr{U}_{g}^{[\bar{X}]} \Psi .
$$

Справедлива и обратная

Tеорема 2. Пусть $(\mathscr{Z}, \mathscr{X}, \pi)$ - квазиклассическое расслоение с калибровочной әруппой $\mathscr{L}$ и выполнен $n .1$ определения 1. Пусть также заданы операторы $\mathscr{U}_{g}^{[\bar{X}]}: C([\bar{X}]) \rightarrow$ $C([\bar{X}])$, сохраняющие норму (9), сильно непрерывные по $g$ при $g=e$, удовлетворяющие свойствам (10) и (11) для калибровочно инвариантных $\alpha$. Тогда при всех $g \in G, X \in \mathscr{X}$ из формуль (8) однозначно определяются операторы $U_{g}\left(u_{g} X \leftarrow X\right): \mathscr{F}_{X} \rightarrow \mathscr{F}_{u_{g}}$. Для них выполняются свойства $2 \mathrm{a})-2$ в) определения 2.

3. Задача исследования представлений групп Ли обычно сводится к изучению представлений алгебр Ли [7]. Рассмотрим инфинитезимальные свойства операторов $\mathcal{U}_{g}^{[\bar{X}]}$ по методу Гординга [8].

Обозначим:

$D_{0}([\bar{X}])$ - множество всех сечений подрасслоения $\left(\mathscr{Z}_{[\bar{X}]},[\bar{X}], \pi\right)$ вида

$$
\Psi_{X}=\int d_{L} g \gamma(g) U_{g}\left(X \leftarrow u_{g^{-1}} X\right) \Phi_{u_{g^{-1}} X}, \quad X \in[\bar{X}],
$$

где $d_{L} g$ - левоинвариантная мера Хаара на группе $G, \gamma(g)$ - гладкая функция на $G$ с компактным носителем, $\Phi \in C_{0}([\bar{X}])$;

$\mathscr{C}_{0}^{\infty}([\bar{X}])$ - множество всех гладких калибровочно инвариантных функций $\alpha_{X}:[\bar{X}] \rightarrow \mathbb{C}$ таких, что множество классов смежности вида $\left\{\bar{h} \in G / G_{0}(\bar{X}) \mid \alpha_{u_{\bar{h}}} \neq 0\right\}$ предкомпактно;

$D([\bar{X}])$ - множество всех линейных комбинаций $v_{[\bar{X}]}\left[\alpha_{1}\right] \Psi_{1}+\cdots+v_{[\bar{X}]}\left[\alpha_{s}\right] \Psi_{s}, \alpha_{i} \in \mathscr{C}_{0}^{\infty}([\bar{X}])$, $\Psi_{i} \in D_{0}([\bar{X}]) ;$

$$
\delta[A] \text { - оператор вида }(\delta[A] \alpha)_{X}=\left.\frac{d}{d \tau}\right|_{\tau=0} \alpha_{u_{\exp A \tau} X} .
$$

ТеОРема 3. Пусть әруппа Ли $G$ действует на квазиклассическом расслоении $(\mathscr{Z}, \mathscr{X}, \pi)$ с калибровочной әруппой $\mathscr{L}$. Тогда

- $D([\bar{X}])$ плотно в $C([\bar{X}])$;

- onepamopbl

$$
\check{H}(A)=\left.i \frac{d}{d \tau}\right|_{\tau=0} \mathscr{U}_{\exp A \tau}^{[\bar{X}]}
$$

определены на $D([\bar{X}])$ и $\check{H}(A): D([\bar{X}]) \rightarrow D([\bar{X}])$;

- справедливъ свойства

$$
\begin{gathered}
\check{H}\left(A_{1}+A_{2}\right) \Psi=\check{H}\left(A_{1}\right) \Psi+\check{H}\left(A_{2}\right) \Psi, \quad \check{H}(\lambda A) \Psi=\lambda \check{H}(A) \Psi, \\
\mathscr{U}_{h}^{[\bar{X}]} \check{H}(A) \mathscr{U}_{h^{-1}}^{[\bar{X}]} \Psi=\check{H}\left(h A h^{-1}\right) \Psi, \quad[\check{H}(A) ; \check{H}(B)] \Psi=i \check{H}([A ; B]) \Psi, \\
i\left[\check{H}(A) ; v_{[\bar{X}]}[\alpha]\right] \Psi=v_{[\bar{X}]}[\delta[A] \alpha] \Psi, \\
-i \delta[A]\langle\Psi, \Psi\rangle=\langle\Psi, \check{H}(A) \Psi\rangle-\langle\check{H}(A) \Psi, \Psi\rangle .
\end{gathered}
$$


ЗАмЕчаниЕ. Предпоследнее из свойств означает, что $\check{H}(A)=H(A ; X)-i \delta[A]$, где $H(A ; X)$ действует в $\mathscr{F}_{X}$.

4. Обратная задача интегрируемости представлений алгебр Ли нетривиальна: известные условия интегрируемости [9]-[12] не очень удобны для использования в квазиклассической теории поля.

Пусть выполнен п. 1 определения 2 , а при каждом $[\bar{X}]$ заданы операторы $\check{H}(A)$ в $C([\bar{X}])$. Наложим на них следующие условия.

УСловиЕ A1. Операторы $\check{H}(A)$ определены на плотной в $C([\bar{X}])$ области $D$.

УСловие А2. Для любых $\Phi, \Psi \in D$ функиия $\langle\Phi, \Psi\rangle$ входит в область определения $\delta[A]$ $u-i \delta[A]\langle\Phi, \Psi\rangle=\langle\Phi, \check{H}(A) \Psi\rangle-\langle\check{H}(A) \Phi, \Psi\rangle$.

УСловие А3. Для любых $\alpha \in \mathscr{C}_{0}^{\infty}([\bar{X}]) u \Psi \in D$

$$
v_{[\bar{X}]}[\alpha] \Psi \in D \quad u \quad\left[\check{H}(A) ; v_{[\bar{X}]}[\alpha]\right] \Psi=-i v_{[\bar{X}]}[\delta[A] \alpha] \Psi .
$$

УСловие А4. Для любъх $\Phi, \Psi \in D$ функиия $\langle\Phi, \check{H}(A) \Psi\rangle$ принадлежит области определения $\delta[B]$.

УСловиЕ А5. Для любых $\Phi, \Psi \in D$ справедливо свойство $\langle\check{H}(A) \Psi ; \check{H}(B) \Phi\rangle-i \delta[A]\langle\Psi, \check{H}(B) \Phi\rangle-\langle\check{H}(B) \Psi, \check{H}(A) \Phi\rangle+i \delta[B]\langle\Psi, \check{H}(A) \Phi\rangle=i\langle\Psi, \check{H}([A ; B]) \Phi\rangle$.

УСловие А6. Пусть $B_{1}, \ldots, B_{n}-$ базис алгебрь Ли и $\Psi^{0} \in D$. Тогда существует решение $\Psi^{t}$ задачи Коши для уравнения

$$
i \frac{d}{d t} \Psi^{t}=\check{H}\left(B_{k}\right) \Psi^{t}
$$

сильно непрерывное по $t$. При этом $\check{H}\left(B_{l}\right) \Psi^{t} \rightarrow \check{H}\left(B_{l}\right) \Psi^{0}$ при $t \rightarrow 0$ в сильном смысле при всех $l$, а функция $\delta[A]\left\langle\Phi, \check{H}\left(B_{k}\right) \Psi_{t}\right\rangle$ непрерывна.

ТЕОРема 4. При условиях $\mathrm{A} 1-\mathrm{A} 6$ однозначно определено действие локальной әруппь Ли $G$ на расслоении $(\mathscr{Z}, \mathscr{X}, \pi)$ такое, что

$$
\left.i \frac{d}{d \tau}\right|_{\tau=0} U_{g(\tau)}\left(X \leftarrow u_{g^{-1}(\tau)} X\right) \Psi_{u_{g^{-1}(\tau)} X}=(\check{H}(A) \Psi)_{X},
$$

$\Psi \in D, g(\tau)=\exp A \tau$.

\section{СПИСОК ЦИТИРОВАННОЙ ЛИТЕРАТУРЫ}

1. Маслов В.П., Шведов О. Ю. Метод комплексного ростка в задаче многих частиц и квантовой теории поля. М.: УРСС, 2000. 2. Маслов В.П. Комплексный метод ВКБ в нелинейных уравнениях. М.: Наука, 1977. 3. Шведов О. Ю. // Матем. заметки. 1999. Т. 65. № 3. С. 437-456. 4. Шведов О. Ю. // Матем. сб. 1999. Т. 190. №10. С. 123-157. 5. Боголюбов Н.Н., Логунов А.А., Оксак А.И., Тодоров И.Т. Общие принципы квантовой теории поля. М.: Наука, 1987. 6. Howland J. // Math. Ann. 1974. V. 207. P. 315-335. 7. Barut A., Raczka R. Theory of Group Representations and Applications. Warszawa: Pol. Sci. Publ., 1977. 8. Garding L. // Proc. Nat. Acad. Sci. USA. 1947. V. 33. P. 331-332. 9. Nelson E. // Ann. Math. 1959. V. 70. P. 572-615. 10. Flato M., Simon J., Snellman H., Sternheimer D. // Ann. Sci. Ecole Norm. Sup. 1972. V. 5. P. 423-434. 11. Simon J. // Comm. Math. Phys. 1972. V. 28. P. 39-46. 12. Flato M., Simon J. // J. Funct. Anal. 1973. V. 13. P. 268-276. 